Acta Crystallographica Section D

\section{Biological Crystallography}

ISSN 0907-4449

\title{
The structure of a novel glucuronoyl esterase from Myceliophthora thermophila gives new insights into its role as a potential biocatalyst
}

The increasing demand for the development of efficient biocatalysts is a consequence of their broad industrial applications. Typical difficulties that are encountered during their exploitation in a variety of processes are interconnected with factors such as temperature, $\mathrm{pH}$, product inhibitors etc. To eliminate these, research has been directed towards the identification of new enzymes that would comply with the required standards. To this end, the recently discovered glucuronoyl esterases (GEs) are an enigmatic family within the carbohydrate esterase (CE) family. Structures of the thermophilic StGE2 esterase from Myceliophthora thermophila (synonym Sporotrichum thermophile), a member of the CE15 family, and its S213A mutant were determined at 1.55 and $1.9 \AA$ resolution, respectively. The first crystal structure of the S213A mutant in complex with a substrate analogue, methyl 4- $O$-methyl- $\beta$-D-glucopyranuronate, was determined at $2.35 \AA$ resolution. All of the three-dimensional protein structures have an $\alpha / \beta$-hydrolase fold with a three-layer $\alpha \beta \alpha$ sandwich architecture and a Rossmann topology and comprise one molecule per asymmetric unit. These are the first crystal structures of a thermophilic GE both in an unliganded form and bound to a substrate analogue, thus unravelling the organization of the catalytic triad residues and their neighbours lining the active site. The knowledge derived offers novel insights into the key structural elements that drive the hydrolysis of glucuronic acid esters.

\section{Introduction}

Lignocellulose in vascular plant cell walls is composed of cellulose, hemicellulose and lignin in relative proportions that vary according to the plant origin (Reddy \& Yang, 2005). A selection of diverse processes, ranging from simple burning to advanced bioconversion, has been applied to access the energy stored in the cell-wall polymers. One of the challenges that researchers face today is to make this process costcompetitive in the biofuels market (Himmel et al., 2007), mainly by overcoming the recalcitrance of biomass. This effort becomes even more demanding owing to the complex structure of the plant cell wall, leading to an increased cost of lignocellulosic conversion (Weng et al., 2008).

Carbohydrate esterases (CEs) are employed as potent biocatalysts for the reduction of the protein load required for the breakdown of lignocellulose to fermentable sugars. One of the recently described CEs, glucuronoyl esterase (GE), has been suggested to play an important role in the dissociation of lignin from hemicellulose and cellulose by cleaving the ester bonds between the aromatic alcohols of lignin and the
Received 6 August 2012 Accepted 10 October 2012

PDB References: StGE2, 4g4g; S213A mutant, 4g4i; S213A mutant in complex with 4-O-methyl- $\beta$-D-glucopyranuronate, $4 \mathrm{~g} 4 \mathrm{j}$
(C) 2013 International Union of Crystallography Printed in Singapore - all rights reserved 
carboxyl groups of 4-O-methyl-D-glucuronic acid residues in glucuronoxylan (Špániková \& Biely, 2006; Ďuranová et al., 2009). GE was first discovered in the wood-rot fungus Schizophyllum commune (Špániková \& Biely, 2006), while the first reported amino-acid sequence was from the Hypocrea jecorina GE Cip2_GE (Li et al., 2007). The latter launched the emerging CE15 family deposited in the continuously updated Carbohydrate-Active Enzymes database (CAZy; http:// www.cazy.org/; Cantarel et al., 2009). To date, six members of this family have been purified and characterized using a series of new synthetic substrates comprising methyl esters of uronic acid and their glycosides. The data obtained showed that the methyl ester of 4-O-methyl-D-glucuronic acid was hydrolyzed more efficiently by all GEs examined compared with the corresponding ester without the 4-O-methyl group (Fig. 1). This finding further supported the potential significance of the methoxy group in substrate recognition (Duranová et al., 2009). To date, the only three-dimensional structure of the CE15 family available is that of Cip2_GE, which confirms the triad arrangement of the putative catalytic residues Ser-HisGlu (Pokkuluri et al., 2011). Here, we report the threedimensional structures of a recombinant thermophilic GE from Myceliophthora thermophila (synonym Sporotrichum thermophile; StGE2) and its S213A mutant determined at 1.55 and $1.90 \AA$ resolution, respectively. We also present for the first time the crystal structure of a GE in complex with a substrate analogue, methyl 4- $O$-methyl- $\beta$-D-glucopyranuronate (MCU), bearing the methoxy group of interest, at 2.35 A resolution.

\section{Materials and methods}

\subsection{Production and purification of recombinant StGE2}

Recombinant $S t$ GE2 and its S213A mutant were produced in Pichia pastoris and subsequently purified using immobilized metal-ion affinity chromatography (IMAC) as described previously. The S213A mutant exhibited a complete loss of enzyme activity towards methyl 4- $O$-methyl-D-glucopyranuronate (Topakas et al., 2010). The homogeneity of the purified samples were assessed by SDS-PAGE using a $12.5 \%$ polyacrylamide gel. A single band corresponding to a molecular mass of $43 \mathrm{kDa}$ was observed, indicating that the protein samples were suitable for crystallization trials.

\subsection{Sequence analysis}

Acknowledging the scarcity of clear crystallographic evidence regarding the putative catalytic site of $S t$ GE2 among

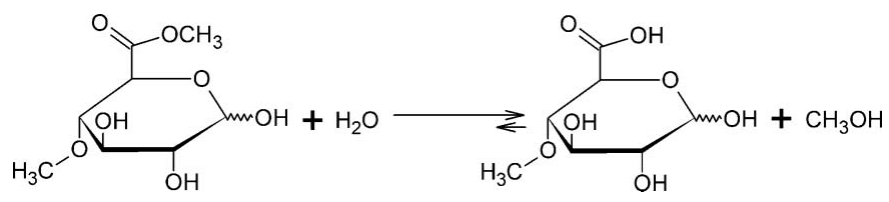

Figure 1

Hydrolysis of methyl 4-O-methyl-D-glucopyranuronate catalyzed by StGE2.
CE15 family members, a broad sequence analysis was performed using Basic Local Alignment Search Tool (BLAST) from the National Center for Biotechnology Information (NCBI) data bank (Altschul et al., 1997). Multiple sequence alignment of homologous enzymes was performed with ClustalW2 (Larkin et al., 2007) on the EBI server (http:// www.ebi.ac.uk/Tools/clustalw2/) and the results were visualized using ESPript2.2 (Gouet et al., 1999; Fig. 2). Secondarystructure assignment and analysis were performed with PROMOTIF (Hutchinson \& Thornton, 1996) as implemented in PDBsum (http://www.ebi.ac.uk/thornton-srv/databases/ pdbsum/; Laskowski et al., 1997) on the EBI server (the results are presented as Supplementary Material ${ }^{\mathbf{1}}$ ).

\subsection{Crystallization and data collection}

The protein samples were concentrated to $20 \mathrm{mg} \mathrm{ml}^{-1}$ in $20 \mathrm{~m} M$ Tris-HCl pH 8.0 buffer prior to crystallization. Initial crystallization conditions for $S t \mathrm{GE} 2$ were established from the commercially available screen The PEGs Suite (Qiagen) using a protein concentration of $10 \mathrm{mg} \mathrm{ml}^{-1}$ in the drop. The crystallization experiments were carried out with the aid of an OryxNano crystallization robot (Douglas Instruments Ltd, UK) using the sitting-drop vapour-diffusion method and 96-well SWISSCI MRC crystallization plates (SWISSCl AG, Zug, Switzerland). Needle-shaped crystals grew at $289 \mathrm{~K}$ and at a $\mathrm{pH}$ ranging from 7.5 to 8.5 in the presence of PEG 2000 MME, 3350, 4000 or 6000 and 25,30 or $35 \%(w / v)$ concentrations of the precipitating agent. Further optimization of both crystal size and quality was carried out and diffracting crystals were grown in $30 \%(w / v)$ PEG $3350,0.1 \mathrm{M}$ Tris- $\mathrm{HCl}$ $\mathrm{pH} 8.0$ to average dimensions of $\sim 10 \times 10 \times 350 \mu \mathrm{m}$ in an orthorhombic habit within one month. X-ray diffraction data were collected at $100 \mathrm{~K}$ using the synchrotron-radiation source at EMBL Hamburg Outstation beamline X13 $(\lambda=0.8123 \AA)$. Prior to data collection, the crystals were transferred into $20 \%(v / v)$ glycerol, which was used as a cryoprotectant. A complete data set was collected to $1.55 \AA$ resolution from a single crystal on a $225 \mathrm{~mm}$ MAR CCD detector using the $D N A$ software package available at beamline X13. Data processing was performed with the $X D S$ package (Kabsch, 2010), followed by data integration and scaling with $S C A L A$ from the CCP4 program suite (Evans, 2006). X-ray diffraction data analysis showed that the $S t \mathrm{GE} 2$ crystal symmetry was consistent with space group $P 2_{1} 2_{1} 2_{1}$, with unit-cell parameters $a=46.0, b=58.7, c=136.2 \AA, \alpha=\beta=\gamma=90^{\circ}$ and one molecule per asymmetric unit. Data-collection statistics for StGE2 are summarized in Table 1.

In the case of the S213A mutant, The PEGs Suite crystallization screen (Qiagen) was again used to identify crystallization conditions. Crystals grew to average dimensions of $\sim 30 \times 30 \times 90 \mu \mathrm{m}$ within one week in the presence of $0.1 \mathrm{M}$ sodium acetate $\mathrm{pH} 4.6,25 \%(v / v)$ PEG $550 \mathrm{MME}$ at $289 \mathrm{~K}$. Diffraction data were collected using a SuperNova in-house

\footnotetext{
${ }^{\mathbf{1}}$ Supplementary material has been deposited in the IUCr electronic archive (Reference: RR5026). Services for accessing this material are described at the back of the journal.
} 
X-ray generator (Agilent Technologies) equipped with a $135 \mathrm{~mm}$ ATLAS CCD detector and a four-circle kappa goniometer at the Institute of Biology, Medicinal Chemistry and Biotechnology, National Hellenic Research Foundation (NHRF; $\mathrm{Cu}$ high-intensity X-ray microfocus source, $\lambda=1.5418 \AA)$ at $100 \mathrm{~K}$ using $15 \%(v / v)$ glycerol as cryoprotectant. Preliminary characterization showed that the S213A mutant crystals belonged to space group $P 2_{1} 22_{1}$, with unit-cell parameters $a=52.0, b=69.6, c=103.8 \AA, \alpha=\beta=\gamma=90^{\circ}$ and one molecule per asymmetric unit. Complete data were collected to $1.9 \AA$ resolution (Table 1) and were processed using the CrysAlis ${ }^{P r o}$ software system (Agilent Technologies) followed by SCALA (Evans, 2006), employing standard protocols for indexing, integration and scaling.

Structural studies of $S t \mathrm{GE} 2$ were performed with the S213A mutant in complex with methyl 4- $O$-methyl-D-glucopyranuronate, which was kindly provided by Dr Peter Biely, Institute of Chemistry of the Slovak Academy of Sciences, Bratislava, Slovakia. This substrate analogue exhibited affinity for $S t \mathrm{GE} 2$, as shown previously (Topakas et al., 2010). Crystals

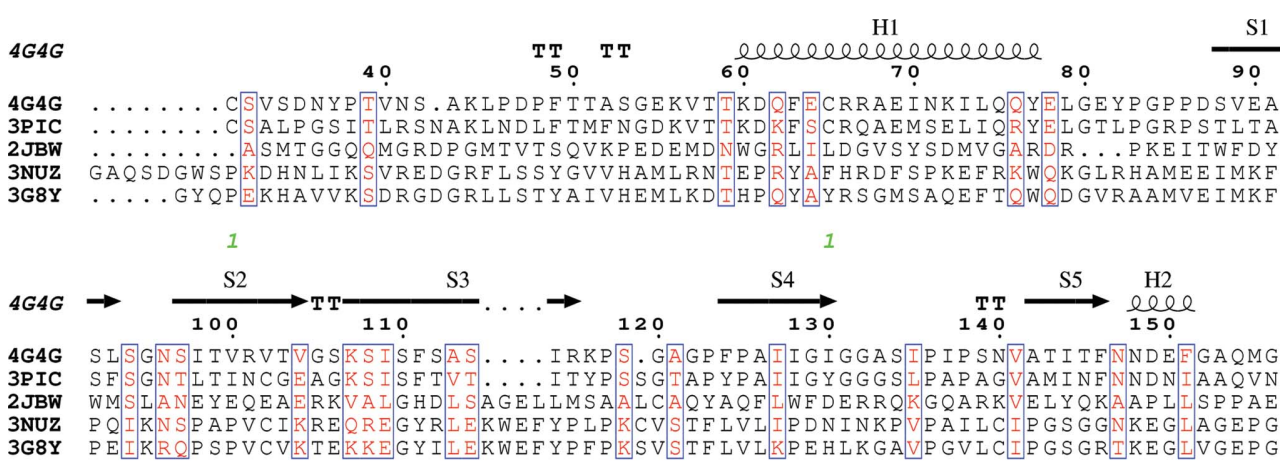

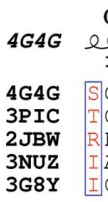

G1

ee eec........ee

$\mathrm{H} 4$

$\mathrm{H} 4 \mathrm{H5}$

180.190

H5

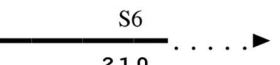

GSRGQGKFYD.

. LFGRDHS

SIT AWRWG

RIII $\operatorname{I} G \mathrm{LE}$

AQASGIDI

210

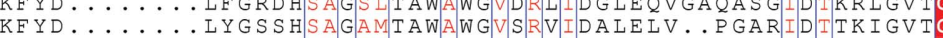

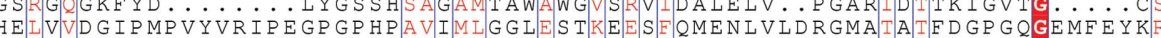
A ARINDRYKDPKLTQALNFVKEGYIAVAVDNPAAGEASDIERYTLGSNYDYDVVSRYLLELG....W

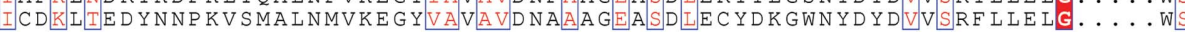

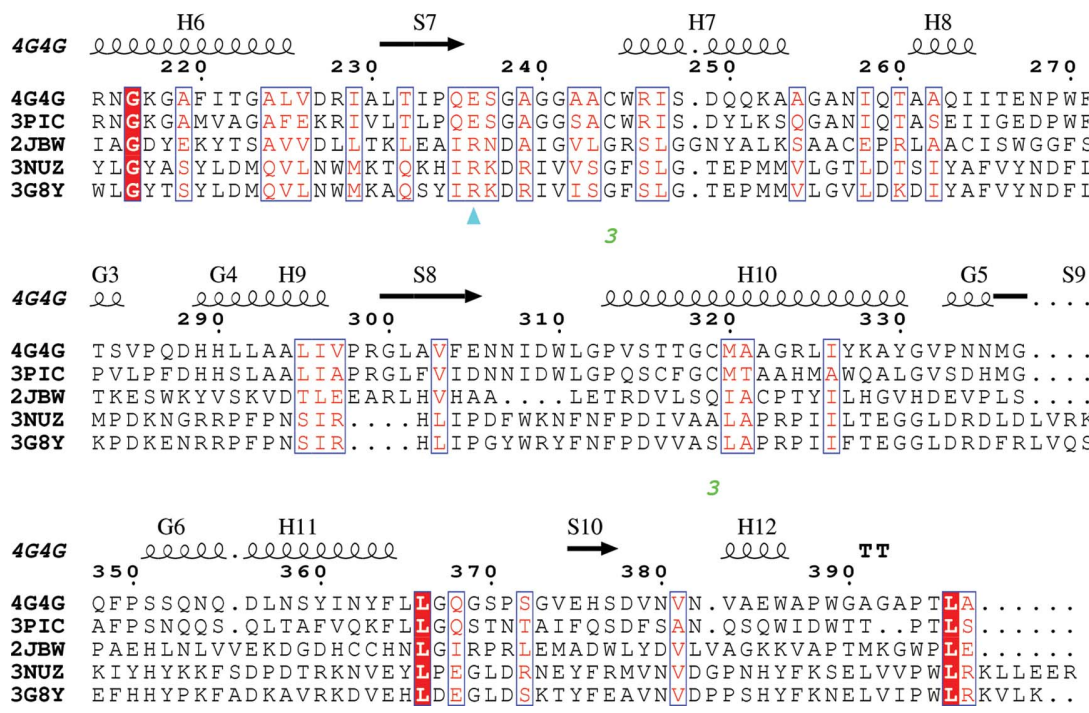

Figure 2

Sequence alignment of $\mathrm{StGE} 2$ (PDB entry $4 \mathrm{~g} 4 \mathrm{~g}$ ) with the best sequence and structural homologues: Cip2_GE (the closest sequence and structural homologue, with $56 \%$ identity over $97 \%$ sequence coverage and a $Z$-score of 59.1; PDB entry 3pic; Pokkuluri et al., 2011), 2,6-dihydroxy-pseudo-oxynicotine hydrolase (the second closest sequence homologue, with $28 \%$ identity over $37 \%$ sequence coverage and a $Z$-score of 16.2; PDB entry 2jbw; Schleberger et al., 2007), putative acetylxylan esterase (a structural homologue with a $Z$-score of 18.8; PDB entry 3nuz; Joint Centre for Structural Genomics, unpublished work) and SusD/ $\mathrm{RagB}$-associated esterase-like protein (a structural homologue with a $Z$-score of 18.8 ; PDB entry $3 \mathrm{~g} 8 \mathrm{y}$; Joint Centre for Structural Genomics, unpublished work). Identical and similar residues are shown in white on a red background and in red on a white background, respectively. The disulfide bonds (numbers in green) and the residues of interest (cyan triangles) are also indicated on the same line. The secondarystructure elements $\alpha$-helices, $3_{10}$-helices, $\beta$-strands and strict $\beta$-turns are denoted H, G, S and TT, respectively. of the S213A mutant were soaked in $5 \mathrm{mM}$ methyl 4-O-methylD-glucopyranuronate dissolved in the mother liquor (Figs. $3 a$ and $3 b$ ) for $1 \mathrm{~h}$. A longer soaking time resulted in crystal deterioration, preventing data collection. The soaked crystals were tested at $100 \mathrm{~K}$ and complete diffraction data were collected to $2.35 \AA$ resolution using the in-house X-ray source at NHRF. The crystal of the complex remained isomorphous to the crystal of the unliganded S213A mutant after soaking, with unit-cell parameters $a=52.1, b=69.8, c=103.9 \AA$, $\alpha=\beta=\gamma=90^{\circ}$. Data processing and scaling was conducted as described for the S213A mutant and the statistics are outlined in Table 1.

\subsection{Structure determination and refinement}

The three-dimensional structure of $\mathrm{StGE} 2$ was determined by molecular replacement using the structure of Cip2_GE (Pokkuluri et al., 2011; PDB entry 3pic) as a starting model and the Phaser crystallographic software (McCoy et al., 2007) as implemented in CCP4 (Winn et al., 2011). The final model was obtained by alternating rounds of anisotropic temperature-factor refinement of all atoms with REFMAC (Murshudov et al., 2011) and manual model building with Coot (Emsley et al., 2010). Water molecules that fulfilled the criteria of forming direct or water-mediated hydrogen-bond interactions with the protein were incorporated into the model also 
Table 1

Diffraction data and refinement statistics for StGE2, the S213A mutant and the S213A mutant in complex with MCU.

Values in parentheses are for the outermost shell.

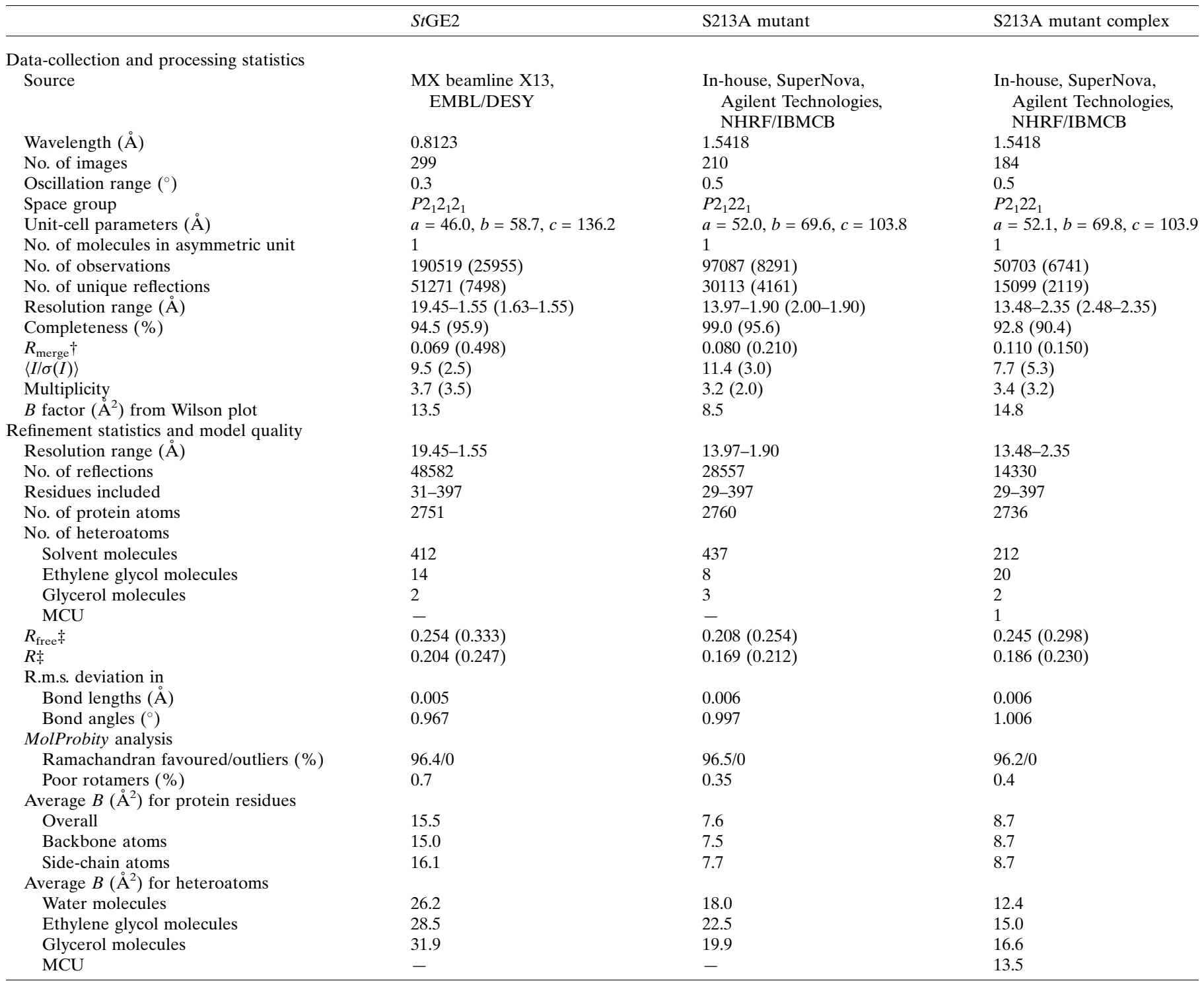

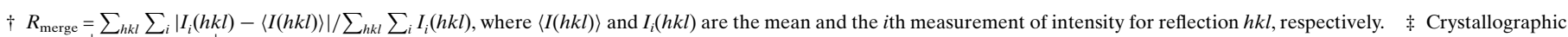

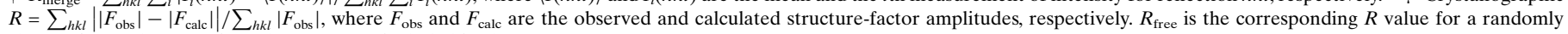
chosen $5 \%$ of the reflections that were not included in the refinement.

using Coot. Visual inspection of the $2 F_{\text {obs }}-F_{\text {calc }}$ and $F_{\text {obs }}-F_{\text {calc }}$ electron-density maps towards the final stages of refinement revealed additional electron density that was attributed to two glycerol and 14 ethylene glycol molecules originating from the cryoprotectant solution and the crystallization medium, respectively. The structure was refined to a final $R$ factor of 0.204 and a final $R_{\text {free }}$ of 0.254 . The refinement statistics are presented in Table 1.

The structure of StGE2 was in turn employed as a starting model for molecular replacement with Phaser (McCoy et al., 2007) using the data for the S213A mutant. Isotropic temperature-factor refinement of all atoms was carried out against experimental data using the same protocol as applied for the native structure. Eight ethylene glycol molecules and three glycerol molecules were included in the model as suggested by both the $2 F_{\text {obs }}-F_{\text {calc }}$ and $F_{\text {obs }}-F_{\text {calc }}$ electrondensity maps. The structure was refined to a final $R$ factor of 0.169 and a final $R_{\text {free }}$ of 0.208 (Fig. 4, Table 1).

Similarly, the structure of the S213A mutant in complex with the synthetic substrate analogue methyl 4-O-methyl-Dglucopyranuronate was determined by employing the mutant structure as a starting model and following a standard protocol for refinement and model building as described above for the S213A StGE2 structure. During the final stages of optimization a portion of extra density was observed at the putative catalytic site of S213A StGE2, suggesting binding of only the $\beta$ anomer of MCU. The MCU model was prepared using the Dundee PRODRG server (http://davapc1.bioch.dundee.ac.uk/ 
prodrg/) and was fitted to the electron density by adjustment of its torsion angles. The model structure was subjected to further refinement (Fig. 3c). In addition, a total of 20 ethylene glycol molecules and two glycerol molecules were incorporated in the final model, which was refined to an $R$ factor of 0.186 and an $R_{\text {free }}$ of 0.245 (Table 1).

\subsection{Structure analysis}

The stereochemistry of the protein residues was validated using PROCHECK (Laskowski et al., 1993) and MolProbity (Chen et al., 2010). The potential hydrogen-bond and van der Waals interactions formed upon the binding of MCU were calculated using the program CONTACT (Winn et al., 2011) applying distance cutoffs of 3.3 and $4.0 \AA$, respectively. Structural superpositions were performed with SUPERPOSE (Winn et al., 2011) and schematic representations of all three crystal structures were prepared with MolSoft (Raush et al., 2009). The programs LIGPLOT (Wallace et al., 1995) and MolScript (Kraulis, 1991) were used to depict the interactions (Figs. 7 and 8), while BobScript (Esnouf, 1997) was employed for schematic representation of the MCU electron-density map (Fig. 3c). Raster3D (Merritt \& Bacon, 1997) was also used to render the images. Structural classification of $S t \mathrm{GE} 2$ folding was performed using the $C A T H$ database of domain structures server. The topology of each structure was extracted using

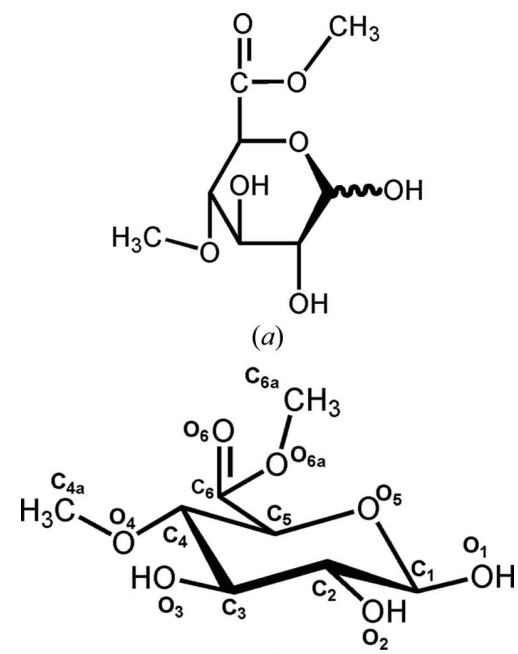

(b)

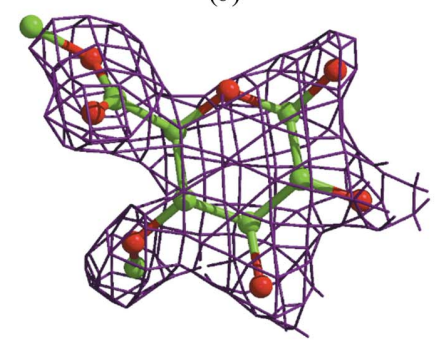

(c)

\section{Figure 3}

(a) Chemical structure of methyl 4-O-methyl-D-glucopyranuronate. (b) The anomer bound to the S213A mutant, also indicating the numbering system used. (c) Schematic representation of the $2 F_{\mathrm{o}}-F_{\mathrm{c}}$ electrondensity map contoured at the $1.0 \sigma$ level of the refined MCU bound in the catalytic cavity of $S t \mathrm{GE} 2$.
PDBsum (http://www.ebi.ac.uk/). Solvent-accessible areas were calculated using the PDBe PISA platform (http:// www.ebi.ac.uk/msd-srv/prot_int/pistart.html; Krissinel \& Henrick, 2007). Structural homologues of $S t \mathrm{GE} 2$ were sought with the aid of the DALI server (Holm \& Rosenström, 2010; Figs. 2 and 5).

\subsection{PDB accession code}

The atomic coordinates and structure factors for the crystal structures of $S t \mathrm{GE} 2$, its S213A mutant and the S213A mutant in complex with methyl $4-O$-methyl- $\beta$-D-glucopyranuronate have been deposited in the Protein Data Bank (http:// www.pdb.org) under accession codes $4 \mathrm{~g} 4 \mathrm{~g}, 4 \mathrm{~g} 4 \mathrm{i}$ and $4 \mathrm{~g} 4 \mathrm{j}$, respectively.

\section{Results and discussion}

\subsection{Sequence analysis}

Analysis of the wild-type $S t \mathrm{GE} 2$ sequence (UniProt code G2QJR6) was performed with BLASTP (Altschul et al., 1997), resulting in a long list of putative conserved domains that all belonged to hypothetical proteins, with the exception of Cip2_GE $(56 \%$ identity and $72 \%$ homology with $92 \%$ coverage). According to the BLASTP results, residues 206227, with amino-acid sequence RLGVTGCSRNGKGAFITGALVD and containing the GXXXXGK motif known as the P-loop motif (Walker et al., 1982), were suggested to have a DUF463 domain architecture (Punta et al., 2012). However, the $e$-value analysis of the results did not provide sufficient evidence to support the existence of the suggested motif. In addition, to the best of our knowledge, esterases involved in

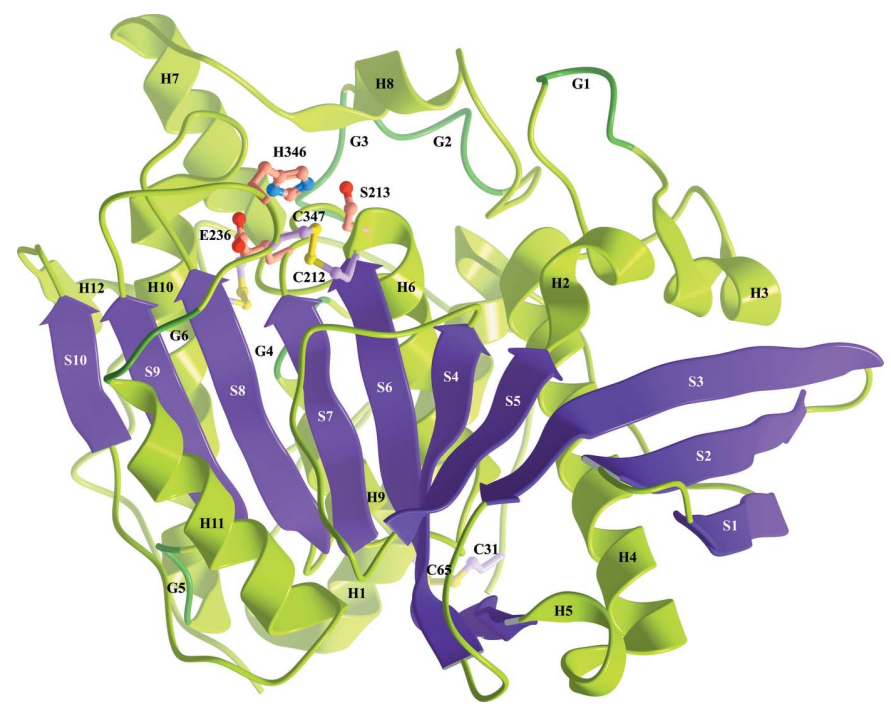

Figure 4

The overall structure of $S t \mathrm{GE} 2$ determined at $1.55 \AA$ resolution. The twisted $\beta$-sheet (shown in violet and labelled $\mathrm{S}$ ) is sandwiched between two layers of $\alpha$-helices (shown in light green and labelled H) and $3_{10}$-helices (shown in dark green and labelled $\mathrm{G}$ ). The catalytic triad residues are indicated in ball-and-stick representation and the disulfide bonds formed are also highlighted. This figure was prepared with MolSoft (Raush et al., 2009). 
the degradation of plant biomass do not require phosphates for activity; therefore, it is unlikely that the presence of this motif has a functional role.

\subsection{Analysis of the StGE2 crystal structures}

Diffracting crystals of $S t \mathrm{GE} 2$ were grown in the orthorhombic lattice, space group $P 2_{1} 2_{1} 2_{1}$, with one molecule per asymmetric unit and the three-dimensional structure was refined to $1.55 \AA$ resolution. A total of 367 amino acids were well defined in the $2 F_{\mathrm{o}}-F_{\mathrm{c}}$ electron-density map and were incorporated in the model. However, there was no density for 14 amino acids at the N-terminus prior to Cys31; therefore, they were excluded from the structure. Similarly, the S213A mutant was prepared by employing previously established protocols (Topakas et al., 2010) to shed light on the structurefunction relationships of the enzyme. Its three-dimensional structure was determined at $1.9 \AA$ resolution from well diffracting crystals grown in the orthorhombic lattice although lacking one twofold axis (space group $P 2_{1} 22_{1}$ ) compared with $S t \mathrm{GE} 2$. The $S t \mathrm{GE} 2$ structure was used as a starting model and two further $\mathrm{N}$-terminal residues were modelled (369 amino acids in total) as suggested by the electron-density maps. However, there was no density for 12 amino acids at the $\mathrm{N}$-terminus prior to Asp29; therefore, they were excluded from the structure. Structural studies of the S213A mutant followed. The S213A mutant complex with methyl 4-O-methylD-glucopyranuronate was formed by soaking preformed crystals and the structure was determined at $2.35 \AA$ resolution. In general, all residues that were included in the final structures lay in allowed regions of the Ramachandran plot. Molecules of ethylene glycol and glycerol, which were used either in the crystallization medium or as a cryoprotectant, were also observed in all three structures, mainly trapped at the interface of the packed monomers in the unit cell and reinforcing the crystal lattice without posing any further modifications (Figs. 4 and 6). However, careful inspection of their binding sites facilitated structure interpretation.

3.2.1. The overall structure of StGE2. StGE2 belongs to the $\alpha / \beta$-hydrolase superfamily and is a serine-type hydrolase; its overall architecture follows the three-layer $\alpha \beta \alpha$-sandwich hydro- lase fold with a Rossmann-fold topology, as revealed by its three-dimensional structure (Fig. 4). However, deviations from the canonical $\alpha / \beta$-hydrolase fold are observed; the canonical fold features a $\beta$-sheet of eight strands in the core of the structure intercalated between two clusters of $\alpha$-helices (four and two $\alpha$-helices bilaterally; Ollis et al., 1992). The $\beta$-sheet is expanded by the insertion of two antiparallel $\beta$-strands at the $\mathrm{N}$-terminus of $\mathrm{St} \mathrm{GE} 2$, resulting in a twisted $\beta$-sheet. The latter is sandwiched between two layers containing a total of 18 helices. Eight $\alpha$-helices and two $3_{10^{-}}$ helices are observed on one side of the $\beta$-sheet instead of two in the canonical fold, while on the opposite side an octet of $\alpha$-helices and $3_{10}$-helices that are equally divided, rather than only four, are recorded. The positions of two of the putative catalytic triad residues, namely Ser213 and Glu236, comply with those suggested by the canonical fold. Detailed analysis of the secondary structure according to PROMOTIF (Hutchinson \& Thornton, 1996) as implemented in PDBsum (Laskowski et al., 1997) is presented in Supplementary Table S1.

The three-dimensional structure of StGE2 with one molecule per asymmetric unit follows the monomeric form that $S t \mathrm{GE} 2$ adopts in solution, as suggested by HPLC gel filtration (data not shown). The latter was confirmed by analysis of the

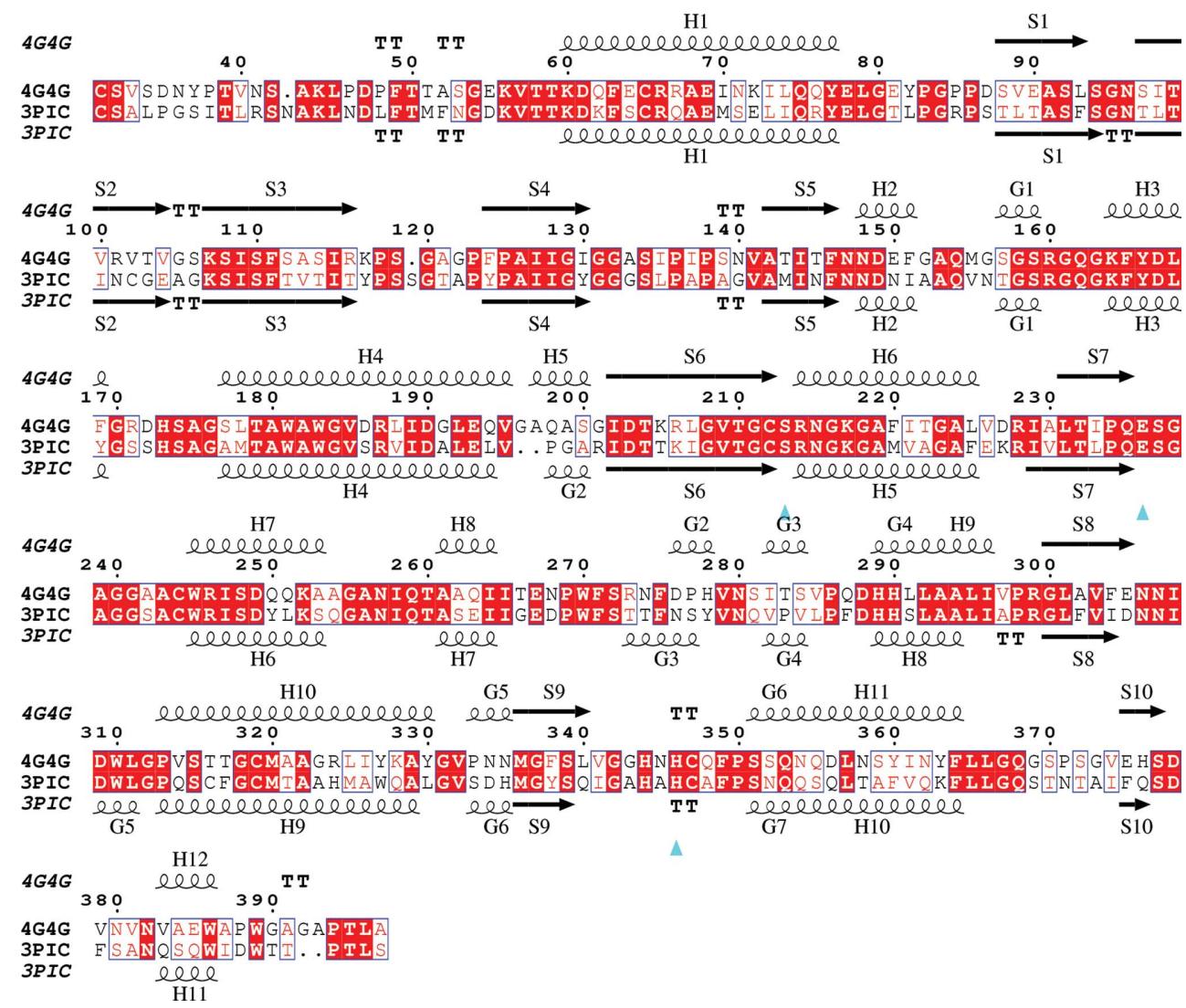

Figure 5

Sequence alignment of StGE2 with Cip2_GE. The secondary-structure elements are highlighted in the consensus line. Identical and similar residues are shown in white on a red background and in red on a white background, respectively. The residues of interest are indicated by cyan triangles in the same line. The secondary-structure elements $\alpha$-helices, $3_{10}$-helices, $\beta$-strands and strict $\beta$-turns are denoted H, G, S and TT, respectively. 
protein interfaces using the PISA server (http://www.ebi.ac.uk/ pdbe/prot_int/pistart.html; Krissinel \& Henrick, 2007), revealing the absence of specific interactions that could result in the formation of stable quaternary structures. The overall structure is stabilized by three disulfide bonds, namely Cys $31-$ Cys65, Cys212-Cys347 and Cys244-Cys319.

Residues Ser213, Glu236 and His346 of StGE2 could be proposed as putative catalytic triad residues since their architecture complies with that proposed for the catalytic

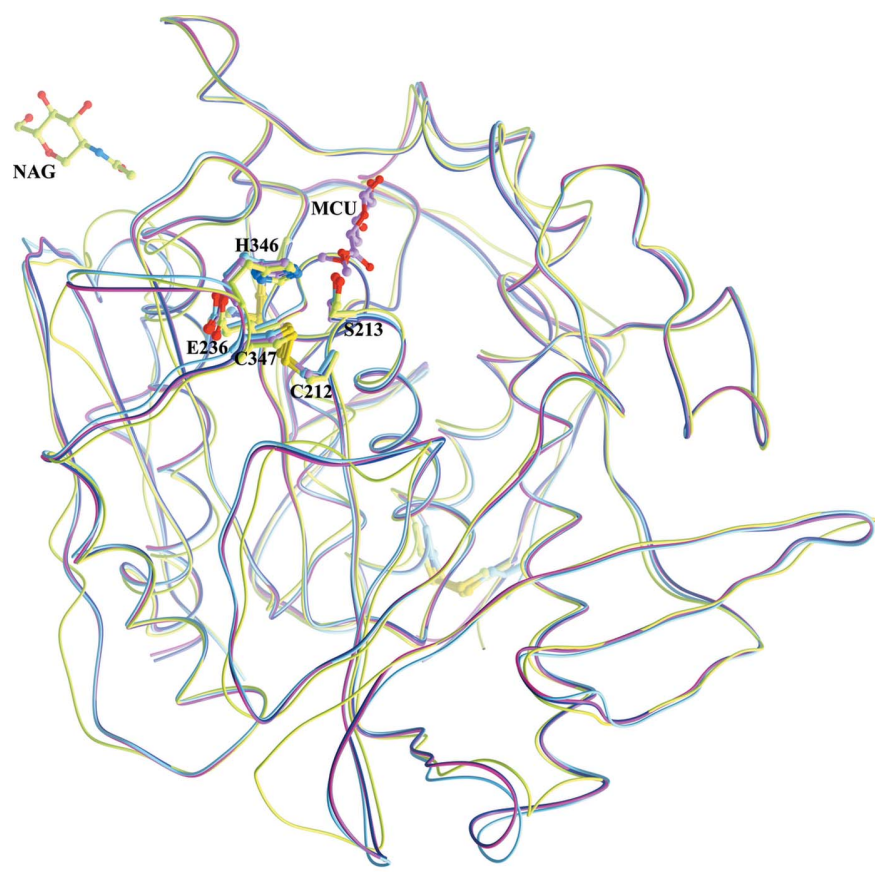

(a)

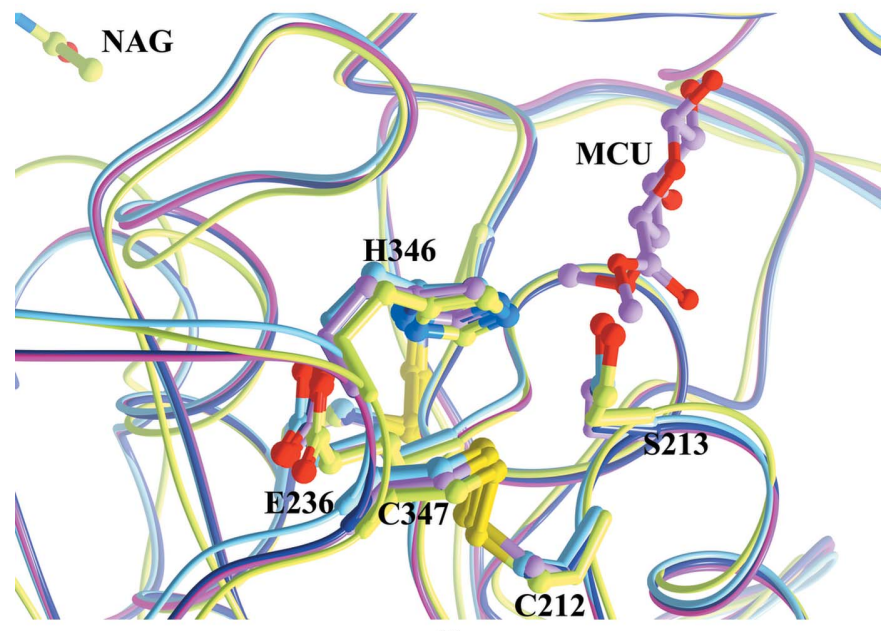

(b)

Figure 6

(a) Superposition of the crystal structures of StGE2 (shown in cyan), the S213A mutant (shown in dark blue), the S213A mutant in complex with MCU (shown in magenta) and the glycosylated ( $N$-acetylglucosamine; NAG) Cip2_GE (shown in lime green; Pokkuluri et al., 2011). The catalytic triad residues are also indicated (the colour scheme follows the relevant structure). The three disulfide bonds that are formed in all structures are highlighted in yellow. (b) Expanded view of the active site indicating the ester bond hydrolyzed by $S t \mathrm{GE} 2$. This figure was prepared with MolSoft (Raush et al., 2009). mechanism of $\alpha / \beta$ hydrolases. Specifically, Ser213 is a nucleophilic serine located between strand S6 and helix H6 forming the so-called 'nucleophilic elbow', with Ramachandran angles lying in the generously allowed regions (Ollis et al., 1992; Nardini \& Dijkstra, 1999; Quevillon-Cheruel et al., 2005). His346 (which acts as an acid/base complementing the nature of the substrate upon binding) bridges strand S9 and helix G6, while Glu236 (which is suggested to adjust the basic character of His346) is situated between strand S7 and helix H7 (Correia et al., 2008). The exact locus and organization of the catalytic triad residues is stabilized by the tight hydrogenbond interactions that are formed between them. The hydroxyl group of Ser213 interacts with His346 NE2, while Glu236 OE1 is hydrogen-bonded to His346 ND1 (at distances of 2.7 and $2.8 \AA$, respectively).

A total of 14 ethylene glycol molecules (present in the crystallization medium) and two glycerol molecules (used as cryoprotectant) were also identified in the structure of $S t \mathrm{GE} 2$. They are predominantly positioned at the interface of the packed monomers, but without providing evidence for specific binding.

3.2.2. Comparison of StGE2 with Cip2_GE from H. jecorina. Comparison of the $S t \mathrm{GE} 2$ structure with that of Cip2_GE, the only known member of the CE15 family (Pokkuluri et al., 2011) for which a structure has been determined to date, showed that the overall structure of the enzymes is homologous and that they share $56 \%$ sequence identity (over the residues observed in the crystal structure, with $97 \%$ coverage) and a $Z$-score of 59 with an r.m.s.d. of $1.1 \AA$ (as determined by the $D A L I$ server for monomer $B$, the closest structural homologue).

However, superposition of the two crystal structures on $\mathrm{C}^{\alpha}$ atoms (Winn et al., 2011) gave an r.m.s.d. of $3.5 \AA$, revealing changes in selected secondary-structure elements and in the $\mathrm{N}$ - and C-termini. Detailed comparison of the two structures showed that the differences detected at sequence level involve either the insertion/deletion of residues leading to alterations in the secondary structure, the presence/absence of a glycosylation site or the packing of the monomers in the crystal. Specifically, the insertion of two residues led to the formation of an $\alpha$-helix (H5, amino acids 197-200) instead of the $3_{10^{-}}$ helix observed in the Cip2_GE structure (Figs. 4, 5 and 6). The resulting structure is stabilized through a water-mediated interaction formed between Gln188 and Glu81' from a symmetry-related molecule. Similar changes were recorded for residues 276-278, which form a 310 -helix (G2) that is shorter in length compared with the Cip2_GE structure. Also, residues 289-292 form a $3_{10}$-helix (G4) and an $\alpha$-helix (H9) rather than only an $\alpha$-helix as in Cip2_GE, and residues 309-

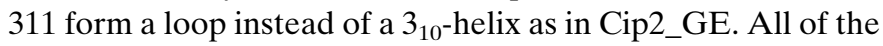
aforementioned changes are observed at the interface of the monomers with symmetry-related molecules in the unit cell. Of these, it is the latter modification involving residues 309311 that is considered to be most significant since it is situated in the vicinity of the catalytic site of $S t \mathrm{GE} 2$. This is accompanied by a difference in the number of residues forming strand S9 (residues 336-340), which contains one more residue 
compared with the corresponding strand in the Cip2_GE structure (Figs. 5 and 6) and adopts a slightly more extended conformation. The succeeding residues belong to a tight turn bearing the catalytic triad residue His346 (see \$3.2.4).

Additional profound changes were observed; however, these could be attributed to shifts imposed by intermolecular interactions of Cip2_GE monomers (with three molecules in the asymmetric unit) as well as interactions with symmetryrelated molecules dictated by crystal packing as in the case of the N- and C-termini (Pokkuluri et al., 2011). Moreover, the residues lining the $\mathrm{H} 12 \alpha$-helix and the preceding loop region are distorted compared with those in the Cip2_GE structure since the $\mathrm{N}$-linked glycosylation sequence motif (Asn- $X$-Ser) and the $N$-acetylglucosamine molecule bound at Asn447 observed in the latter are missing in StGE2 (Asn- $X$-Ala is the corresponding motif in $\mathrm{StGE2}$; Fig. 6).

Previous studies of the effect of temperature on $S t \mathrm{GE} 2$ stability showed that $S t \mathrm{GE} 2$ remains active at $323 \mathrm{~K}$ for at

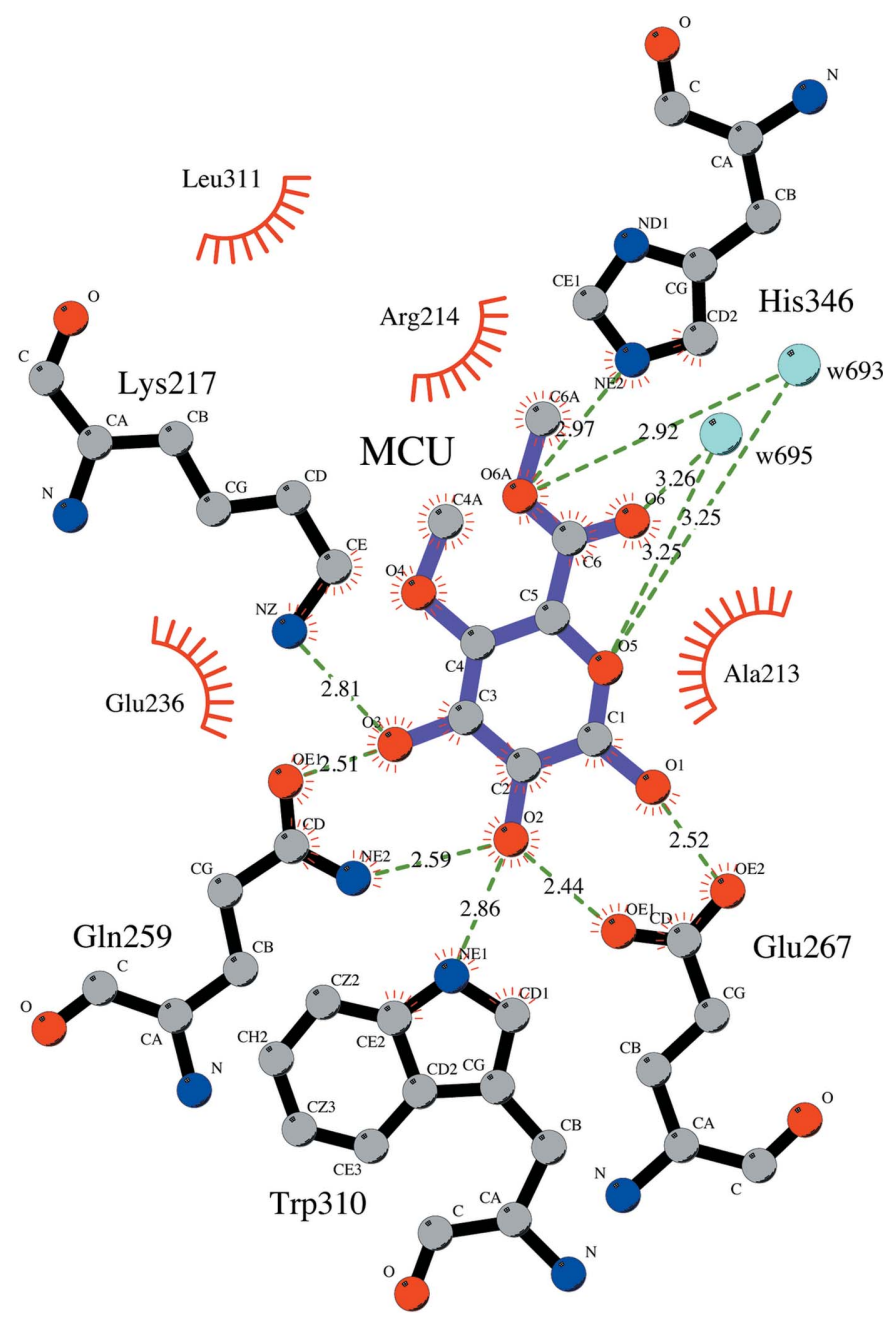

Figure 7

LIGPLOT diagram of MCU interacting with the active-site residue lying in the vicinity. The substrate-analogue bonds are shown in purple, while bonds of the residues lining the site are shown in black. Hydrogen bonds are shown as black dashed lines with distances indicated in $\AA$. Additional residues forming van der Waals interactions with MCU are represented by red semicircles with radiating spokes. least $24 \mathrm{~h}$, maintains more than $70 \%$ of its activity at 333$343 \mathrm{~K}$ and exhibits half-lives of $22 \mathrm{~h}$ at $328 \mathrm{~K}$ and $0.5 \mathrm{~h}$ at $333 \mathrm{~K}$ (Topakas et al., 2010), while Cip2_GE is only quite stable at $313 \mathrm{~K}$, with half-lives of 10 and $2 \mathrm{~min}$ at 323 and $333 \mathrm{~K}$, respectively (Li et al., 2007). A comparative analysis of the structures of the thermophilic StGE2 and its mesophilic homologue Cip2_GE was performed to monitor the differences in the structural determinants that could affect their thermostabilities. The typical indicators employed to assess protein thermostability are rather diverse. At the sequence level the protein composition of prolines has been examined, taking into account their limited allowed configurations and the restrictions that they impose on the preceding residue (Prajapati et al., 2007). The primary structure of StGE2 contains 22 proline residues compared with 17 in Cip2_GE. Moreover, the number of thermolabile residues such as Met, Cys, Asn and Gln was considered. StGE2 contains 48 thermolabile residues compared with 54 in Cip2_GE, as is observed in the majority of thermophilic proteins when compared with their mesophile homologues (Kumar et al., 2000). The ratio of Arg/(Arg+Lys) has also been used as a indicator for this purpose. StGE2 exhibits an increased Arg/ (Arg+Lys) ratio compared with Cip2_GE (0.56 and 0.5, respectively; Mrabet et al., 1992). Similarly, electrostatic interactions are among the structural features which are known to be shared by thermostable proteins. The structures of $S t \mathrm{GE} 2$ and Cip2_GE present the same number of salt bridges and cation $-\pi$ interactions, as calculated using the ESBRI server (Costantini et al., 2008) and CaPTURE (Gallivan \& Dougherty, 1999).

3.2.3. The overall structures of the unliganded and bound forms of the S213A mutant. The S213A mutant was prepared following a previously developed protocol (Topakas et al., 2010) to shed light on the structure-function relationships of the enzyme and the putative role of the nucleophilic Ser in the substrate-recognition/catalytic mechanism. Determination of its three-dimensional structure at $1.9 \AA$ resolution revealed that overall it remained unchanged with reference to the StGE2 crystal structure except for some areas involved in crystallographic symmetry interactions. Superposition of the S213A mutant atomic coordinates with those of StGE2 on $\mathrm{C}^{\alpha}$ atoms showed that the two structures differed only slightly by an r.m.s.d. of $0.4 \AA$. Similarly, the crystal structure of the S213A mutant in complex with MCU indicated that the substrate analogue bound at the catalytic site of the enzyme without affecting the overall structure (r.m.s.d. on $\mathrm{C}^{\alpha}$ atoms of 0.40 and $0.12 \AA$ with reference to the $S t \mathrm{GE} 2$ and the S213A mutant structures, respectively; Fig. 6). Ethylene glycol and glycerol molecules were also bound in both structures as in the case of $S t \mathrm{GE} 2$. Of these, two ethylene glycol molecules were located in the active-site region of the S213A mutant structure (Table 1, Fig. 6; see \$3.2.4).

3.2.4. StGE2 catalytic site. Overall, the catalytic site in the monomeric three-dimensional structure of StGE2 lies on the surface of the molecule; it is mostly exposed to the solvent and is not affected by symmetry-related packing interactions. All residues lining the putative catalytic triad face the solvent, 
Table 2

Direct hydrogen bonds and water-mediated hydrogen-bond interactions of MCU in the catalytic pocket of S213A StGE2.

There are a total of seven direct hydrogen-bond interactions

\begin{tabular}{lllc}
\hline Atom & Protein atom & Distance $(\AA)$ & Angle $\left(^{\circ}\right)$ \\
\hline O1 & Glu267 OE2 & 2.5 & 119.9 \\
O2 & Gln259 NE2 & 2.6 & 127.1 \\
& Glu267 OE1 & 2.4 & 119.2 \\
& Trp310 NE1 & 2.9 & 169.8 \\
O3 & Lys217 NZ & 2.8 & 0.0 \\
& Gln259 OE1 & 2.5 & 127.6 \\
O5 & Wat693 O & 3.3 & - \\
& Wat695 O & 3.3 & - \\
O6 & Wat695 O & 3.3 & - \\
O6a & His346 NE2 & 3.0 & - \\
& Wat693 O & 2.9 & - \\
& Arg214 NH1 & 3.2 & \\
\hline
\end{tabular}

Table 3

van der Waals interactions of MCU in the catalytic pocket of S213A StGE2.

There are a total of 59 van der Waals interactions.

\begin{tabular}{lll}
\hline $\begin{array}{l}\text { Substrate } \\
\text { atom }\end{array}$ & Protein atoms & $\begin{array}{l}\text { No. of } \\
\text { contacts }\end{array}$ \\
\hline C1 & Glu267 OE1, OE2 & 2 \\
C2 & Gln259 OE1, NE2; Glu267 OE1, OE2, CD; & 6 \\
& Trp310 NE1 & \\
C3 & Lys217 NZ; Gln259 OE1 & 2 \\
C4 & Lys217 NZ & 1 \\
C4a & Ala213 CA, CB; Lys217 NZ; Glu236 O; Leu311 CD2; & 7 \\
& Wat670; Wat712 & \\
C5 & Wat693; Wat695; Wat712 & 3 \\
C6 & Ala213 CB; His346 NE2; Wat693; Wat695 & 4 \\
C6a & Ala213 CB; Arg214 NH1; His346 NE2, CD2; Wat694; & 6 \\
& Wat693 & 3 \\
O1 & Glu267 CD, OE1; Wat695 & 6 \\
O2 & Gln259 OE1, CD; Glu267 CD, OE2; Trp310 CD1, CE22 & 4 \\
O3 & Lys217 CE; Gln259 CD, NE2; Wat670 & 6 \\
O4 & Ala213 CA, CB, C; Arg214 N; Lys217 CE, NZ \\
O6 & Ala213 CB; Arg214 CB, CG, CD, NH1, N & 6 \\
O6a & Ala213 CB; His346 CD2; Wat694 & 3 \\
\hline
\end{tabular}

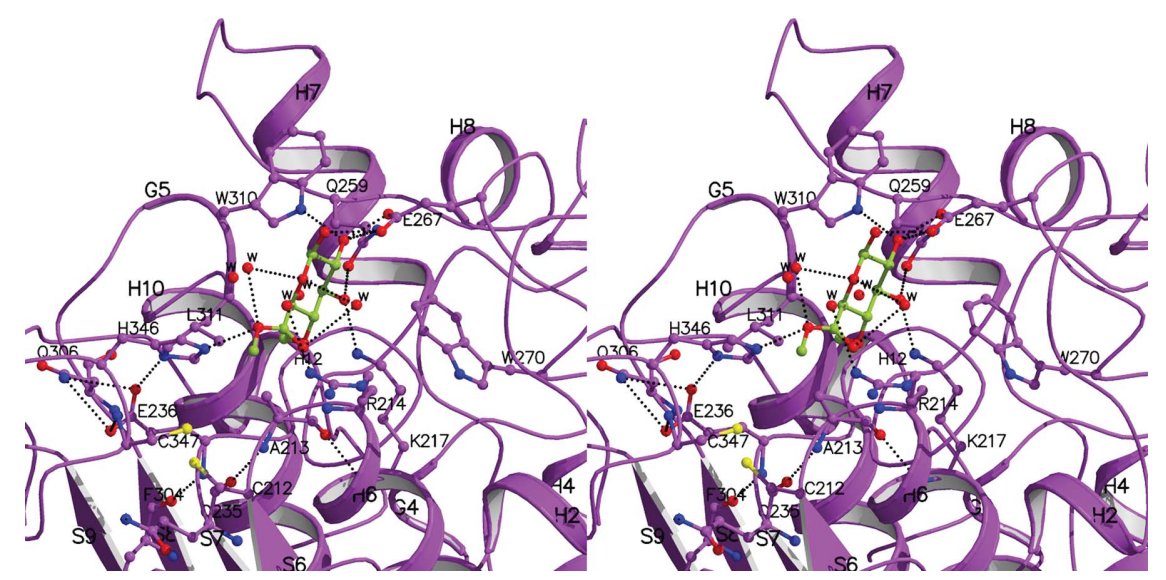

Figure 8

Stereo diagram of the interactions between the S213A mutant and MCU bound at the catalytic pocket as well as the hydrogen bonds formed by the catalytic triad residues. The side chains and backbone atoms of protein residues involved in ligand binding are shown in ball-and-stick representation. Water molecules (w) are depicted as spheres and hydrogen-bond interactions are shown as dotted lines. This figure was prepared with MolScript (Kraulis, 1991) and was rendered with Raster3D (Merritt \& Bacon, 1997). with accessible surface areas for Ser213, Glu236 and His346 of 27.1, 2.7 and $75.7 \AA^{2}$, respectively. The catalytic residues Ser213 and Glu236 are not occluded by symmetry-related polypeptide chains, except for those in the vicinity of His 346. The $S t \mathrm{GE} 2$ structure suggests that strands $\mathrm{S} 9$ and $\mathrm{S} 7$ shape the active site, coordinating His 346 and Glu236 towards forming the catalytic triad with Ser213 (Fig. 4). The catalytic triad residues are involved in a network of hydrogen bonds that contribute to the rigid architecture of the catalytic pocket. Focusing on the nucleophilic Ser213, its hydroxyl group interacts directly with His346 NE2, which in turn forms a hydrogen bond between its ND1 atom and Glu236 OE1. The setting is complemented by additional direct and watermediated polar interactions involving residues Arg214, Gly216, Lys217, Gln235, Phe304 and Asn306 (Supplementary Table S2). This mode of interaction provides structural evidence for the nucleophilic role of Ser213, while His346 is expected to act as an acid-base complementing the nature of the substrate upon binding and Glu236 is expected to play a role as a regulator of the basic character of histidine (Correia et al., 2008).

Mutation of Ser213 to Ala showed that the absence of the hydroxyl group did not affect the active-site architecture. The hydrogen bonds formed between the Ala213 backbone atoms ( $\mathrm{N}$ and $\mathrm{O}$ ) and nearby residues, as well as the contacts with His346 and Glu236, were maintained. The loop bearing His346 (residues $344-350$ ) is subjected to shifts ranging from $\sim 0.5$ to $\sim 1.0 \AA$ in the S213A mutant owing to crystal-packing interactions. Despite these shifts, the position of His346 in the catalytic site is preserved (minor shifts of $\sim 0.5 \AA$ of all atoms). The disulfide bond formed between Cys 347 and Cys 212 further enhances the rigidity of the catalytic site by bridging the two adjacent catalytic triad residues (His346 and Ser213, respectively). Additional electron density for two ethylene glycol molecules EDO406 and EDO408 was identified in the S213A mutant structure at the same site. The latter lay at the entrance of the cavity, forming five potential hydrogen bonds to the neighbouring residues Lys217 NZ, Gln259 OE1 and NE2, Glu267 OE2 and Trp310 NE1 (Supplementary Fig. S1). This finding reveals that the catalytic cavity is exposed to the solvent and has the potential to accommodate the substrate by forming favourable binding interactions (Supplementary Tables S3, S4 and S5).

With the aim of advancing our knowledge of the structure-function relationship of $S t$ GE2 and elucidating the underlying mechanism of enzyme action, structural studies of the S213A mutant in complex with the substrate analogue MCU were performed. The results revealed that the substrate analogue bound at the catalytic site, forming an extended network of interactions (seven direct and four indirect hydrogen bonds as well as 59 van der Waals 
interactions) with the active-site residues (Tables 2 and 3 , Figs. 7 and 8). The residues participating in the hydrogen-bond network were those observed in the S213A mutant structure with ethylene glycol together with His346 NE2. Two O atoms of the glucopyranose ring, $\mathrm{O} 2$ and $\mathrm{O} 3$, are aligned with the positions of the ethylene glycol hydroxyl groups shifted by $\sim 0.7$ and $\sim 0.2 \AA$, respectively (Figs. 7 and 8 , Supplementary Fig. S1).

Specifically, the substrate analogue rests in the cavity using the peripheral hydroxyl group $\mathrm{O}$ atoms of the glucopyranose ring as an anchor to promote its binding. Both the Gln259 and Glu267 side-chain atoms are hydrogen-bonded to $\mathrm{O} 1, \mathrm{O} 2$ and O3 of the sugar. The conformation of the Glu267 side chain is altered [rotation of its $\left(\chi_{1}, \chi_{3}\right)$ dihedral angles by $\left(\sim 74^{\circ}\right.$, $\left.\sim 127^{\circ}\right)$ ] compared with both the S213A mutant and the StGE2 structures, favouring tight binding of the substrate analogue. Trp310 NE1 is also in close contact with O2, while Lys 217 NZ interacts with O3. An additional polar contact is formed between Lys $217 \mathrm{NZ}$ and $\mathrm{O} 4$ at a distance of $3.4 \AA$. O5 completes this hydrogen-bonding arrangement by taking part in water-mediated interactions with Arg214 NH1 (through water molecules Wat693 and Wat695). O6a, which pertains to the ester bond to be cleaved, is hydrogen-bonded to the NE2 atom of the imidazole ring of His346; this contact contributes to the suggestion that the position of the catalytic triad is rather concrete and the pocket is crafted to impose a 'ready for nucleophilic attack' orientation of the substrate regardless of the absence of the nucleophile. To this end, comparison of the StGE2 crystal structure with the complex structure demonstrates that the Ser213 OG atom is indeed facing the ester bond at a distance of $\sim 2.2 \AA$.

The van der Waals interactions of the substrate analogue mainly concern the same palette of residues as those involved in the aforementioned hydrogen bonds. Among these, attention is drawn to the backbone $\mathrm{N}$ atom of Arg214 and its counterparts, $\mathrm{O} 4$ of the methoxy moiety and O6 of the ester group (at a distance of $\sim 3.4 \AA$ ), which is also hydrogenbonded to Ser213 OG, implying that Arg214 is positioned to be part of the so-called oxyanion hole (Figs. 7 and 8).

Superposition of the structures of the unliganded S213A mutant and the complex showed that MCU binds at the same position as previously occupied by one of the two ethylene glycol molecules bound in the catalytic pocket in the unliganded structure. In particular, EDO408 mimics the positions of the $\mathrm{C} 2, \mathrm{O} 2, \mathrm{C} 3$ and $\mathrm{O} 3$ atoms of the glupyranose ring, while EDO406 lies at the vicinity of the site. The complex was prepared using preformed crystals of the S213A mutant; therefore, it appears that the substrate analogue displaced the ethylene glycol molecules from the catalytic pocket upon binding, explaining the quality of the electron density attributed to the ligand (Figs. $3 c$ and 7 and Supplementary Fig. S1).

\section{Conclusions}

The crystal structures of a recombinant novel thermophilic $\mathrm{GE}$ and of unliganded and bound forms of its S213A mutant were determined at $1.55,1.9$ and $2.35 \AA$ resolution, respec- tively. $S t \mathrm{GE} 2$ is a member of the $\alpha / \beta$-hydrolase superfamily and its overall structure follows the three-layer $\alpha \beta \alpha$-sandwich hydrolase fold with a Rossmann topology. Three disulfide bonds, one of which is situated at the entrance to the catalytic pocket, contribute to the rigidity of the structure and the active-site architecture. Mapping of the catalytic site using a methyl 4- $O$-methyl- $\beta$-D-glucopyranuronate substrate analogue revealed that the catalytic triad residues, namely Ser213, Glu236 and His346, participate in a concrete 'ready for nucleophilic attack' configuration. This is partially coordinated by strands $\mathrm{S} 6, \mathrm{~S} 7$ and $\mathrm{S} 9$ of the $\beta$-sheet, which drive His346 and its triad counterparts into an orientation that makes substrate recognition possible. The complex structure also unveiled the inherent flexibility of residues shaping the pocket, such as Glu267, which alters its side-chain conformation to accommodate the substrate analogue. The setting is complemented by the Arg214 backbone N atom of the socalled oxyanion hole that lies opposite the ester and methoxy group and is proposed to stabilize the tetrahedral intermediate during catalysis. The methoxy group, comprising atoms $\mathrm{O} 4$ and $\mathrm{C} 4 \mathrm{a}$, might also play an additional role to the catalytic triad residues in substrate recognition by enhancing binding $v i a$ an increased number of van der Waals interactions formed with the residues lining the site (Table 3).

StGE2 and Cip2_GE are the only characterized GEs for which three-dimensional structures have been determined to date. Direct comparison of the two enzymes brings to light the biochemical and structural determinants that promote $S t \mathrm{GE} 2$ as a more suitable target that could be further explored for potential biotechnological applications in comparison to Cip2_GE. These comprise the thermophilicity of the former, the accessibility of the catalytic triad residues and the crafting of the active site towards substrate recognition, as well as its monomeric form in both the crystal and solution. We believe that the X-ray structure of StGE2 and the complex structure of its S213A mutant have uncovered the fingerprint of substrate binding to a GE for the first time, appraising its potential to be exploited as a model for developing tailor-made effective biocatalysts. The next step in this direction is a thorough investigation of $S t \mathrm{GE} 2$ by site-directed mutagenesis to delineate its functional role and to develop a prototype for bioconversion.

This research was co-financed by the European Union (European Social Fund - ESF) and Greek national funds through the Operational Program 'Education and Lifelong Learning' of the National Strategic Reference Framework (NSRF) - Research Funding Program Heracleitus II, Investing in Knowledge Society through the European Social Fund. This work was also supported by the FP7 Capacities coordination and support actions REGPOT-2008-1 No. 230146 'EUROSTRUCT' and REGPOT-2009-1 No. 245866 'ARCADE'; the research leading to these results also received funding from the European Community's Seventh Framework Programme (FP7/2007-2013) under grant agreement No. 226716. We would like to thank Dr Peter Biely, Institute of 
Chemistry of the Slovak Academy of Sciences (Bratislava, Slovakia), who kindly provided methyl 4-O-methyl-D-glucopyranuronate for binding studies. The EMBL staff at PX beamline X13 at the DORIS storage ring, DESY, Hamburg and especially Dr Spyros Chatziefthimiou are also acknowledged for their help during data collection.

\section{References}

Altschul, S. F., Madden, T. L., Schäffer, A. A., Zhang, J., Zhang, Z., Miller, W. \& Lipman, D. J. (1997). Nucleic Acids Res. 25, 3389-3402.

Cantarel, B. L., Coutinho, P. M., Rancurel, C., Bernard, T., Lombard, V. \& Henrissat, B. (2009). Nucleic Acids Res. 37, D233-D238.

Chen, V. B., Arendall, W. B., Headd, J. J., Keedy, D. A., Immormino, R. M., Kapral, G. J., Murray, L. W., Richardson, J. S. \& Richardson, D. C. (2010). Acta Cryst. D66, 12-21.

Correia, M. A., Prates, J. A., Brás, J., Fontes, C. M., Newman, J. A., Lewis, R. J., Gilbert, H. J. \& Flint, J. E. (2008). J. Mol. Biol. 379, 64-72.

Costantini, S., Colonna, G. \& Facchiano, A. M. (2008). Bioinformation, 3, 137-138.

Ďuranová, M., Hirsch, J., Kolenová, K. \& Biely, P. (2009). Biosci. Biotechnol. Biochem. 73, 2483-2487.

Emsley, P., Lohkamp, B., Scott, W. G. \& Cowtan, K. (2010). Acta Cryst. D66, 486-501.

Esnouf, R. M. (1997). J. Mol. Graph. Model. 15, 132-134.

Evans, P. (2006). Acta Cryst. D62, 72-82.

Gallivan, J. P. \& Dougherty, D. A. (1999). Proc. Natl Acad. Sci. USA, 96, 9459-9464.

Gouet, P., Courcelle, E., Stuart, D. I. \& Métoz, F. (1999). Bioinformatics, 15, 305-308.

Himmel, M. E., Ding, S.-Y., Johnson, D. K., Adney, W. S., Nimlos, M. R., Brady, J. W. \& Foust, T. D. (2007). Science, 315, 804-807.

Holm, L. \& Rosenström, P. (2010). Nucleic Acids Res. 38, W545W549.

Hutchinson, E. G. \& Thornton, J. M. (1996). Protein Sci. 5, 212-220.

Kabsch, W. (2010). Acta Cryst. D66, 125-132.

Kraulis, P. J. (1991). J. Appl. Cryst. 24, 946-950.

Krissinel, E. \& Henrick, K. (2007). J. Mol. Biol. 372, 774-797.

Kumar, S., Tsai, C.-J. \& Nussinov, R. (2000). Protein Eng. 13, 179-191.

Larkin, M. A., Blackshields, G., Brown, N. P., Chenna, R., McGettigan, P. A., McWilliam, H., Valentin, F., Wallace, I. M., Wilm, A., Lopez, R., Thompson, J. D., Gibson, T. J. \& Higgins, D. G. (2007). Bioinformatics, 23, 2947-2948.
Laskowski, R. A., Hutchinson, E. G., Michie, A. D., Wallace, A. C., Jones, M. L. \& Thornton, J. M. (1997). Trends Biochem. Sci. 22, 488-490.

Laskowski, R. A., MacArthur, M. W., Moss, D. S. \& Thornton, J. M. (1993). J. Appl. Cryst. 26, 283-291.

Li, X.-L., Špániková, S., de Vries, R. P. \& Biely, P. (2007). FEBS Lett. 581, 4029-4035.

McCoy, A. J., Grosse-Kunstleve, R. W., Adams, P. D., Winn, M. D., Storoni, L. C. \& Read, R. J. (2007). J. Appl. Cryst. 40, 658-674.

Merritt, E. A. \& Bacon, D. J. (1997). Methods Enzymol. 277, 505-524.

Mrabet, N. T. et al. (1992). Biochemistry, 31, 2239-2253.

Murshudov, G. N., Skubák, P., Lebedev, A. A., Pannu, N. S., Steiner, R. A., Nicholls, R. A., Winn, M. D., Long, F. \& Vagin, A. A. (2011). Acta Cryst. D67, 355-367.

Nardini, M. \& Dijkstra, B. W. (1999). Curr. Opin. Struct. Biol. 9, 732-737.

Ollis, D. L., Cheah, E., Cygler, M., Dijkstra, B., Frolow, F., Franken, S. M., Harel, M., Remington, S. J., Silman, I., Schrag, J., Sussman, J. L., Verschueren, K. H. G. \& Goldman, A. (1992). Protein Eng. 5, 197-211.

Pokkuluri, P. R., Duke, N. E. C., Wood, S. J., Cotta, M. A., Li, X.-L., Biely, P. \& Schiffer, M. (2011). Proteins, 79, 2588-2592.

Prajapati, R. S., Das, M., Sreeramulu, S., Sirajuddin, M., Srinivasan, S., Krishnamurthy, V., Ranjani, R., Ramakrishnan, C. \& Varadarajan, R. (2007). Proteins, 66, 480-491.

Punta, M. et al. (2012). Nucleic Acids Res. 40, D290-D301.

Quevillon-Cheruel, S., Leulliot, N., Graille, M., Hervouet, N., Coste, F., Bénédetti, H., Zelwer, C., Janin, J. \& Van Tilbeurgh, H. (2005). Protein Sci. 14, 1350-1356.

Raush, E., Totrov, M., Marsden, B. D. \& Abagyan, R. (2009). PLoS One, 4, e7394.

Reddy, N. \& Yang, Y. (2005). Trends Biotechnol. 23, 22-27.

Schleberger, C., Sachelaru, P., Brandsch, R. \& Schulz, G. E. (2007). J. Mol. Biol. 367, 409-418.

Špániková, S. \& Biely, P. (2006). FEBS Lett. 580, 4597-4601.

Topakas, E., Moukouli, M., Dimarogona, M., Vafiadi, C. \& Christakopoulos, P. (2010). Appl. Microbiol. Biotechnol. 87, 17651772.

Walker, J. E., Saraste, M., Runswick, M. J. \& Gay, N. J. (1982). EMBO J. 1, 945-951.

Wallace, A. C., Laskowski, R. A. \& Thornton, J. M. (1995). Protein Eng. 8, 127-134.

Weng, J.-K., Li, X., Bonawitz, N. D. \& Chapple, C. (2008). Curr. Opin. Biotechnol. 19, 166-172.

Winn, M. D. et al. (2011). Acta Cryst. D67, 235-242. 